\title{
FUZZY STABILITY FOR A CLASS OF QUADRATIC FUNCTIONAL EQUATIONS
}

\author{
GiLJUN HAN*
}

\begin{abstract}
In this paper, we investigate the following form of a certain class of quadratic functional equations and its fuzzy stability.

$f(k x+y)+f(k x-y)=f(x+y)+f(x-y)-2\left(1-k^{2}\right) f(x)$
\end{abstract}

where $k$ is a fixed rational number with $k \neq 1,-1,0$.

\section{Introduction and preliminaries}

In 1964, S. M. Ulam proposed the following stability problem(cf [13]) : "Let $G_{1}$ be a group and $G_{2}$ a metric group with the metric $d$. Given a constant $\delta>0$, does there exist a constant $c>0$ such that if a mapping $f: G_{1} \longrightarrow G_{2}$ satisfies $d(f(x y), f(x) f(y))<c$ for all $x, y \in G_{1}$, then there exists a unique homomorphism $h: G_{1} \longrightarrow G_{2}$ with $d(f(x), h(x))<$ $\delta$ for all $x \in G_{1}$ ?"

In 1941, D. H. Hyers [5] answered this problem under the assumption that the groups $G_{1}$ and $G_{2}$ are Banach spaces. Aoki [1] and Rassias [12] generalized the Hyers' result. Rassias [12] solved the generalized Hyers-Ulam stability of the functional inequality

$$
\|f(x+y)-f(x)-f(y)\| \leq \epsilon\left(\|x\|^{p}+\|y\|^{p}\right)
$$

for some $\epsilon \geq 0$ and $p$ with $p<1$ and all $x, y \in X$, where $f: X \longrightarrow Y$ is a function between Banach spaces. The paper of Rassias[12] has provided a lot of influence in the development of what we call the generalized Hyers-Ulam stability or Hyers-Ulam-Rassias stability of functional equations. A generalization of the Rassias theorem was obtained by

Received December 24, 2013; Accepted January 16, 2014.

2010 Mathematics Subject Classification: Primary 39B52, 39B72, 46 S40.

Key words and phrases: fuzzy normed space, quadratic functional equation, fuzzy stability.

The present research was conducted by the research fund of Dankook university in 2012. 
Gavruta [4] by replacing the unbounded Cauchy difference by a general control function in the sprit of Rassias approach. Kim, Han and Shim [8] investigated Hyers-Ulam stability for a class of quadratic functional equations via a typical form

$$
\begin{aligned}
& f(a x+b y)+f(a x-b y)-2 a^{2} f(x)-2 b^{2} f(y) \\
& +c[f(x+y)+f(x-y)-2 f(x)-2 f(y)]=0 .
\end{aligned}
$$

In this paper, we consider the fuzzy version of stability for the class of quadratic fuctional equations

$$
f(k x+y)+f(k x-y)=f(x+y)+f(x-y)-2\left(1-k^{2}\right) f(x)
$$

in the fuzzy normed space setting. The concept of fuzzy norm on a linear space was introduced by Katsaras [7] in 1984. Bag and Samanta [2], following Cheng and Mordeson [3], gave an idea of a fuzzy norm in such a manner that the corresponding fuzzy metric is of Kramosil and Michalek type [9]. In 2008, A. K. Mirmostafaee and M. S. Moslehian $[10,11]$ used the definition of a fuzzy norm in [2] to obtain a fuzzy version of stability for the additive functional equation

$$
f(x+y)=f(x)+f(y),
$$

and the quadratic functional equation

$$
f(x+y)+f(x-y)=2 f(x)+2 f(y) .
$$

We call a solution of (1.1) an additive mapping or briefly additive and a solution of (1.2) is called a quadratic mapping or briefly quadratic.

Now, we introduce the following functional equation for fixed rational number $k$ with $k \neq 1,-1,0$ :

$$
f(k x+y)+f(k x-y)=f(x+y)+f(x-y)-2\left(1-k^{2}\right) f(x)
$$

in a fuzzy normed space. It is easy to see that the function $f(x)=p x^{2}$ is a solution of the functional equation (1.3). Now, we recall the following definition for a fuzzy normed space given in [12] and the fundamental concepts:

Definition 1.1. Let $X$ be a real linear space. A function $N: X \times$ $\mathbb{R} \longrightarrow[0,1]$ is called a fuzzy norm on $X$ if for all $x, y \in X$ and all $s, t \in \mathbb{R}$,

(N1) $N(x, t)=0$ for $t \leq 0$;

(N2) $x=0$ if and only if $N(x, t)=1$ for all $t>0$;

(N3) $N(c x, t)=N\left(x, \frac{t}{|c|}\right)$ if $c \neq 0$;

(N4) $N(x+y, s+t) \geq \min \{N(x, s), N(y, t)\}$; 
(N5) $N(x, \cdot)$ is a nondecreasing function of $\mathbb{R}$ and $\lim _{t \longrightarrow \infty} N(x, t)=1$; (N6) for $x \neq 0, N(x, t)$ is continuous on $\mathbb{R}$.

In this case, the pair $(X, N)$ is called a fuzzy normed linear space.

The examples of fuzzy norms and properties of fuzzy normed linear spaces are given in $[10,11]$.

Definition 1.2. Let $(X, N)$ be a fuzzy normed linear space. A sequence $\left\{x_{n}\right\}$ in $X$ is said to be convergent if there exists an $x \in X$ such that $\lim _{n \longrightarrow \infty} N\left(x_{n}-x, t\right)=1$ for all $t>0$. In this case, $x$ is called the limit of the sequence $\left\{x_{n}\right\}$ and we denote it by $N-\lim _{n \longrightarrow \infty} x_{n}=x$.

Definition 1.3. Let $(X, N)$ be a fuzzy normed vector space. A sequence $\left\{x_{n}\right\}$ in $\mathrm{X}$ is said to be Cauchy if for each $\epsilon>0$ and each $t>0$ there is an $m \in N$ such that for all $n \geq m$ and all $p>0$, we have $N\left(x_{n+p}-x_{n}, t\right)>1-\epsilon$.

It is well known that every convergent sequence in a fuzzy normed space is Cauchy. If each Cauchy sequence is convergent, then the fuzzy norm is said to be complete and the fuzzy complete normed space is called a fuzzy Banach space.

\section{Solution of (1.3)}

In this section, we investigate solutions of (1.3). In Theorem 2.2, we conclude that any solution of (1.3) is quadratic if $k$ is a rational number with $k \neq 1,-1,0$. We start with the following lemma.

Lemma 2.1. If $f: X \longrightarrow Y$ satisfies (1.3) for $k \neq 0$, then the following equation holds.

$$
\begin{aligned}
& \left(-1+\frac{1}{k^{2}}\right)[f(2 x+y)+f(2 x-y)] \\
& -2\left(-1+\frac{1}{k^{2}}\right)[f(x+y)+f(x-y)] \\
& +\left(-1+\frac{1}{k^{2}}\right) f(y)+\left(-1+\frac{1}{k^{2}}\right) f(-y) \\
& +4\left(-1+\frac{1}{k^{2}}\right) f(x)-2\left(-1+\frac{1}{k^{2}}\right) f(2 x)=0
\end{aligned}
$$

for all $x, y \in X$.

Proof. Putting $x=0=y$ in (1.3), we get $f(0)=0$. Letting $y=0$ in (1.3), we have

$$
f(k x)=k^{2} f(x)
$$


for all $x \in X$.

Replacing $y$ by $x+y$ in (1.3), we have

$$
\begin{aligned}
& f((k+1) x+y))+f((k-1) x-y))-[f(2 x+y)+f(-y)] \\
& =2\left(k^{2}-1\right) f(x)
\end{aligned}
$$

for all $x, y \in X$ and letting $y=-y$ in (2.3), we have

$$
\begin{aligned}
& f((k+1) x-y))+f((k-1) x+y))-[f(2 x-y)+f(y)] \\
& =2\left(k^{2}-1\right) f(x)
\end{aligned}
$$

for all $x, y \in X$.

Replacing $x$ and $y$ by $x+\frac{1}{k} y$ and $x$ in (1.3) respectively, we have

$$
\begin{aligned}
& f((k+1) x+y))+f((k-1) x+y))-\left[f\left(2 x+\frac{1}{k} y\right)+f\left(\frac{1}{k} y\right)\right] \\
& =2\left(k^{2}-1\right) f\left(x+\frac{1}{k} y\right)
\end{aligned}
$$

for all $x, y \in X$ and letting $y=-y$ in (2.5), we have

$$
\begin{aligned}
& f((k+1) x-y))+f((k-1) x-y))-\left[f\left(2 x-\frac{1}{k} y\right)+f\left(-\frac{1}{k} y\right)\right] \\
& =2\left(k^{2}-1\right) f\left(x-\frac{1}{k} y\right)
\end{aligned}
$$

for all $x, y \in X$. By (2.3), (2.4), (2.5), and (2.6), we have

$$
\begin{aligned}
& -[f(2 x+y)+f(2 x-y)+f(y)+f(-y)] \\
& +\frac{1}{k^{2}}[f(y)+f(-y)]+\frac{1}{k^{2}}[f(2 a x+y)+f(2 a x-y)] \\
& =4\left(k^{2}-1\right) f(x)-2\left(1-\frac{1}{k^{2}}\right)[f(a x+y)+f(a x-y)]
\end{aligned}
$$

for all $x, y \in X$. By (1.3) and (2.7), we have

$$
\begin{aligned}
& -[f(2 x+y)+f(2 x-y)+f(y)+f(-y)]+\frac{1}{k^{2}}[f(y)+f(-y)] \\
& +\frac{1}{k^{2}}\left[f(2 x+y)+f(2 x-y)-2 f(2 x)-2 f(y)-2 k^{2} f(2 x)-2 f(y)\right] \\
& =4\left(k^{2}-1\right) f(x) \\
& -2\left(1-\frac{1}{k^{2}}\right)\left[f(x+y)+f(x-y)-2 f(x)-2 f(y)-2 k^{2} f(x)-2 f(y)\right]
\end{aligned}
$$

for all $x, y \in X$. Now, just simplifying this equation, we can get the result. 
Theorem 2.2. Suppose that $f: X \longrightarrow Y$ satisfies (1.3). Then $f$ is quadratic.

Proof. Suppose that $f$ satisfies (1.3). Then by (2.1) in lemma 2.1, we have

$$
\begin{aligned}
& \frac{1}{k^{2}}\left(1-k^{2}\right)[f(2 x+y)+f(2 x-y)] \\
& =\frac{1}{k^{2}}\left(1-k^{2}\right)[2 f(x+y)+2 f(x-y)-4 f(x)+2 f(2 x)-f(y)-f(-y)]
\end{aligned}
$$

for all $x, y \in X$. Hence by [6], $f$ is quadratic-cubic. Now since $f(k x)=$ $k^{2} f(x), f$ is quadratic.

\section{Fuzzy stability for the functional equation (1.3)}

Let $X$ be a real linear space, $(Y, N)$ be a fuzzy Banach space and $\left(Z, N^{\prime}\right)$ be a fuzzy normed space, respectively. As a matter of convenience, for a given mapping $f: X \longrightarrow Y$, we use the abbreviation

$D f(x, y)=f(k x+y)+f(k x-y)-\left[f(x+y)+f(x-y)-2\left(1-k^{2}\right) f(x)\right]$

for all $x, y \in X$. Now we will prove fuzzy version of stability for the functional equation (1.3).

Theorem 3.1. Let $f: X \longrightarrow Y$ be a mapping such that $f(0)=0$ and

$$
N(D f(x, y), t) \geq N^{\prime}(\phi(x, y), t)
$$

for all $x, y \in X$ and all $t>0$. Let $\phi: X^{2} \longrightarrow Z$ be a function and $r$ be a real number such that $0<|r|<k^{2}$ such that

$$
N^{\prime}(\phi(k x, k y), t) \geq N^{\prime}(r \phi(x, y), t)
$$

for all $x, y \in X$ and all $t>0$. Then there exists a unique quadratic mapping $Q: X \longrightarrow Y$ such that the inequality

$$
N(Q(x)-f(x), t) \geq N^{\prime}\left(\frac{1}{2\left(k^{2}-|r|\right)} \phi(x, 0), t\right)
$$

holds for all $x \in X$ and all $t>0$.

Proof. Inequality (3.1) is equivalent to the following :

$$
\begin{aligned}
& N\left(f(k x+y)+f(k x-y)-\left[f(x+y)+f(x-y)+2\left(1+k^{2}\right) f(x)\right], t\right) \\
& \geq N^{\prime}(\phi(x, y), t)
\end{aligned}
$$


for all $x, y \in X$ and all $t>0$. By (3.2) and (N3), we have

$$
N^{\prime}\left(\phi\left(k^{n} x, k^{n} y\right), t\right) \geq N^{\prime}\left(r^{n} \phi(x, y), t\right)=N^{\prime}\left(\phi(x, y), \frac{t}{|r|^{n}}\right)
$$

for all $x, y \in X$ and all $t>0$ and so by (3.5), we have

$$
N^{\prime}\left(\phi\left(k^{n} x, k^{n} y\right), \quad|r|^{n} t\right) \geq N^{\prime}(\phi(x, y), t)
$$

for all $x, y \in X$ and all $t>0$. Letting $y=0$ in (3.4), by (N3), we have

$$
N\left(\frac{f(k x)}{k^{2}}-f(x), \frac{t}{2 k^{2}}\right) \geq N^{\prime}(\phi(x, 0), t)
$$

for all $x \in X$ and all $t>0$. By (3.2), (3.6), (3.7), and (N3), we have

$N\left(\frac{f\left(k^{n+1} x\right)}{k^{2(n+1)}}-\frac{f\left(k^{n} x\right)}{k^{2 n}}, \frac{|r|^{n} t}{2 k^{2(n+1)}}\right) \geq N^{\prime}\left(\phi\left(k^{n} x, 0\right),|r|^{n} t\right) \geq N^{\prime}(\phi(x, 0), t)$

for all $x \in X$, all $t>0$ and all positive integers $n$. Hence by (3.8) and (N4), for any $x \in X$, we have

$$
\begin{aligned}
& N\left(\frac{f\left(k^{n} x\right)}{k^{2 n}}-f(x), \sum_{i=0}^{n-1} \frac{|r|^{i} t}{2 k^{2(i+1)}}\right) \\
& =N\left(\sum_{i=0}^{n-1}\left[\frac{f\left(k^{i+1} x\right)}{k^{2(i+1)}}-\frac{f\left(k^{i} x\right)}{k^{2 i}}\right], \sum_{i=0}^{n-1} \frac{|r|^{i} t}{2 k^{2(i+1)}}\right) \\
& \geq \min \left\{N\left(\frac{f\left(k^{i+1} x\right)}{k^{2(i+1)}}-\frac{f\left(k^{i} x\right)}{k^{2 i}}, \frac{|r|^{i} t}{2 k^{2(i+1)}}\right) \mid 0 \leq i \leq n-1\right\} \\
& \geq N^{\prime}(\phi(x, 0), t)
\end{aligned}
$$

for all $x \in X$, all $t>0$ and all positive integers $n$. So for any $x \in X$, we have

$$
\begin{aligned}
& N\left(\frac{f\left(k^{m+p} x\right)}{k^{2(m+p)}}-\frac{f\left(k^{m} x\right)}{k^{2 m}}, \sum_{i=m}^{m+p-1} \frac{|r|^{i} t}{2 k^{2(i+1)}}\right) \\
& =N\left(\sum_{i=m}^{m+p-1}\left[\frac{f\left(k^{i+1} x\right)}{k^{2(i+1)}}-\frac{f\left(k^{i} x\right)}{k^{2 i}}\right], \sum_{i=m}^{m+p-1} \frac{|r|^{i} t}{2 k^{2(i+1)}}\right) \\
& \geq \min \left\{N\left(\frac{f\left(k^{i+1} x\right)}{k^{2(i+1)}}-\frac{f\left(k^{i} x\right)}{k^{2 i}}, \frac{|r|^{i} t}{2 k^{2(i+1)}}\right) \mid m \leq i \leq m+p-1\right\} \\
& \geq N^{\prime}(\phi(x, 0), t)
\end{aligned}
$$


for all $x \in X$, all $t>0$ and all positive integers $m, p$. Thus, by (3.10) and (N3), for any $x \in X$, we have

$$
N\left(\frac{f\left(k^{m+p} x\right)}{k^{2(m+p)}}-\frac{f\left(k^{m} x\right)}{k^{2 m}}, t\right) \geq N^{\prime}\left(\phi(x, 0), \frac{t}{\sum_{i=m}^{m+p-1} \frac{\mid r^{i}}{2 k^{2(i+1)}}}\right)
$$

for all $x \in X$, all $t>0$ and all positive integers $m$ and $p$. Since $\sum_{i=0}^{\infty} \frac{|r|^{i}}{2 k^{2(i+1)}}$ is convergent, $\lim _{m \longrightarrow \infty} \frac{t}{\sum_{i=m}^{m+p-1} \frac{|r|^{i}}{2 k^{2(i+1)}}}=\infty$ and so $\left\{\frac{f\left(k^{m} x\right)}{k^{2 m}}\right\}$ is a Cauchy sequence in $(Y, N)$. Since $(Y, N)$ is a fuzzy Banach space, there is a mapping $Q: X \longrightarrow Y$ defined by

$$
\begin{aligned}
& Q(x)=N-\lim _{n \longrightarrow \infty} \frac{f\left(k^{n} x\right)}{k^{2 n}} \text { or } \\
& \lim _{n \longrightarrow \infty} N\left(Q(x)-\frac{f\left(k^{n} x\right)}{k^{2 n}}, t\right)=1, t>0
\end{aligned}
$$

for all $x \in X$. Moreover by (3.9), we have

$$
N\left(\frac{f\left(k^{n} x\right)}{k^{2 n}}-f(x), t\right) \geq N^{\prime}\left(\phi(x, 0), \frac{t}{\sum_{i=0}^{n-1} \frac{|r|^{i}}{2 k^{2(i+1)}}}\right)
$$

for all $x \in X$, all $t>0$ and all positive integers $m, p$. Let $\epsilon$ be a real number $0<\epsilon<1$. Then, by (3.12), (3.13), and (N4), we have

$$
\begin{aligned}
& N(Q(x)-f(x), t) \\
& \geq \min \left\{N\left(Q(x)-\frac{f\left(k^{n} x\right)}{k^{2 n}}, \epsilon t\right), N\left(\frac{f\left(k^{n} x\right)}{k^{2 n}}-f(x),(1-\epsilon t)\right)\right\} \\
& \geq N^{\prime}\left(\phi(x, 0), \frac{(1-\epsilon) t}{\sum_{i=0}^{n-1} \frac{|r|^{i}}{2 k^{2(i+1)}}}\right) \\
& \geq N^{\prime}\left(\phi(x, 0), 2(1-\epsilon)\left(k^{2}-|r|\right) t\right)
\end{aligned}
$$

for sufficiently large positive integer $n$, all $x \in X$, and all $t>0$. Since $N(x, \cdot)$ is continuous on $\mathbb{R}$, we get

$$
N(Q(x)-f(x), t) \geq N^{\prime}\left(\phi(x, 0), 2\left(k^{2}-|r|\right) t\right)
$$

for all $x \in X$ and all $t>0$ and so we have (3.3).

By (3.2) and (N5), we have 


$$
\begin{aligned}
& N\left(\frac{D f\left(k^{n} x, k^{n} y\right)}{k^{2 n}}, t\right) \\
& \geq N^{\prime}\left(\phi\left(k^{n} x, k^{n} y\right), k^{2 n} t\right) \\
& \geq N^{\prime}\left(\phi(x, y), \frac{k^{2 n}}{|r|^{n}} t\right)
\end{aligned}
$$

for all $x, y \in X$ and all $t>0$. Since $\lim _{n \longrightarrow \infty} N^{\prime}\left(\phi(x, y), \frac{k^{2 n}}{|r|^{n}} t\right)=1$, by (3.12), (3.16), and (N4), we have

$$
\begin{aligned}
& N(D Q(x, y), t) \\
& \geq \min \left\{N\left(D Q(x, y)-\frac{D f\left(k^{n} x, k^{n} y\right)}{k^{2 n}}, \frac{t}{2}\right), N\left(\frac{D f\left(k^{n} x, k^{n} y\right)}{k^{2 n}}, \frac{t}{2}\right)\right\} \\
& \geq N\left(\frac{D f\left(k^{n} x, k^{n} y\right)}{k^{2 n}}, \frac{t}{2}\right) \\
& \geq N^{\prime}\left(\phi(x, y), \frac{k^{2 n}}{2|r|^{n}} t\right), t>0
\end{aligned}
$$

for sufficiently large $n$, all $x, y \in X$ and all $t>0$, because

$$
\lim _{n \longrightarrow \infty} N\left(Q(x, y)-\frac{D f\left(k^{n} x, k^{n} y\right)}{k^{2 n}}, t\right)=1
$$

for all $x \in X$ and all $t>0$. Since $\lim _{n \longrightarrow \infty} N^{\prime}\left(\phi(x, y), \frac{k^{2 n}}{|r|^{n}} t\right)=1$, $N(D Q(x, y), t)=1$ for all $t>0$ and so, by (N2), $D Q(x, y)=0$ for all $x, y \in X$. By Theorem 2.2, $Q$ is quadratic.

To prove the uniquness of $Q$, let $Q_{1}: X \longrightarrow Y$ be another quadratic mapping satisfying (3.3). Then for any $x \in X$ and a positive integer $n$, $Q_{1}\left(k^{n} x\right)=k^{2 n} Q_{1}(x)$ and so by $(3.13)$,

$$
\begin{aligned}
& N\left(Q(x)-Q_{1}(x), t\right) \\
& \geq \min \left\{N\left(\frac{Q\left(k^{n} x\right)}{k^{2 n}}-\frac{f\left(k^{n} x\right)}{k^{2 n}}, \frac{t}{2}\right), N\left(\frac{Q_{1}\left(k^{n} x\right)}{k^{2 n}}-\frac{f\left(k^{n} x\right)}{k^{2 n}}, \frac{t}{2}\right)\right\} \\
& \geq N^{\prime}\left(\phi\left(k^{n} x, 0\right), k^{2 n}\left(k^{2}-|r|\right) t\right) \\
& \geq N^{\prime}\left(\phi(x, 0), \frac{k^{2 n}\left(k^{2}-|r|\right) t}{|r|^{n}}\right)
\end{aligned}
$$


holds for all $x \in X$, all positive integer $n$, and all $t>0$. Since $|r|<k^{2}$, $\lim _{n \longrightarrow \infty} N^{\prime}\left(\phi(x, 0), \frac{k^{2 n}\left(k^{2}-|r|\right) t}{|r|^{n}}\right)=1$ and so $Q(x)=Q_{1}(x)$ for all $x \in X$.

Similar to Theorem 3.1, we have the following theorem :

TheOREm 3.2. Let $f: X \longrightarrow Y$ be a mapping such that $f(0)=0$ and

$$
N(D f(x, y), t) \geq N^{\prime}(\phi(x, y), t)
$$

for all $x, y \in X$ and all $t>0$. Let $\phi: X^{2} \longrightarrow Z$ be a function and $r$ be a real number such that $0<k^{2}<|r|$ such that

$$
N^{\prime}\left(\phi\left(\frac{x}{k}, \frac{y}{k}\right), t\right) \geq N^{\prime}\left(\frac{1}{r} \phi(x, y), t\right)
$$

for all $x, y \in X$ and all $t>0$. Then there exists a unique quadratic mapping $Q: X \longrightarrow Y$ such that the inequality

$$
N(Q(x)-f(x), t) \geq N^{\prime}\left(\frac{1}{2\left(|r|-k^{2}\right)} \phi(x, 0), t\right)
$$

holds for all $x \in X$ and all $t>0$.

We can use Theorem 3.1 and Theorem 3.2 to get a classical result in the framework of normed spaces.

For any normed space $(X,\|\cdot\|)$, the mapping $N_{X}: X \times \mathbb{R} \longrightarrow[0,1]$, defined by

$$
N_{X}(x, t)= \begin{cases}0, & \text { if } t \leq 0 \\ \frac{t}{t+|| x \mid}, & \text { if } t>0\end{cases}
$$

a fuzzy norm on $X$. Using this, we have the following corollary :

Corollary 3.3. Let $f: X \longrightarrow Y$ be a mapping such that $f(0)=0$ and

$$
\|D f(x, y)\| \leq\|x\|^{p}\|y\|^{p}+\|x\|^{2 p}+\|y\|^{2 p} .
$$

for all $x, y \in X$, a fixed rational number $k$ and a fixed real number $p$ such that $|k|>1$ and $0<p<1$ or $|k|<1$ and $p>1$. Then there exists a unique quadratic mapping $Q: X \longrightarrow Y$ such that the inequality

$$
\|Q(x)-f(x)\| \leq \frac{\|x\|^{2 p}}{\left|k^{2}-k^{2 p}\right|}
$$

holds for all $x \in X$. 


\section{References}

[1] T. Aoki, On the stability of the linear transformation in Banach space, J. Math. Soc. Japan 2 (1950), 64-66.

[2] T. Bag and S. K. Samanta, Finite dimensional fuzzy normed linear spaces, J. Fuzzy Math. 3 (2003), 687-705.

[3] S. C. Cheng and S. N. Mordeson, Fuzzy linear operator and Fuzzy normed linear spaces, Bull. Calcutta Math. Soc. 86 (1994), 429-436.

[4] P. Gavruta, A generalization of the Hyers-Ulam-Rassias of approximatel additive mappings, J. Math. Anal. 184 (1994), 431-436.

[5] D. H. Hyers, On the stability of the linear functional equations, Proc. Nat. Acad. Sci. USA 27 (1941), 222-224.

[6] K. W. Jun, H. M. Kim, and I. S. Chang, On the Hyers-Ulam stability of an Euler-Lagrange type cubic functional equation, J. Comp. Anal. and Appl. 7 (2005), 21-33.

[7] A. K. Katsaras, Fuzzy topological vector space II Fuzzy Sets System , Fuzzy Sets System. 12 (1984), 143-154.

[8] C. I. Kim, G. J. Han, and S. A. Shim, Hyers-Ulam stability for a class of quadratic functional equations via a typical form, Abs. Appl. Anal. 2013 (2013), 1-8.

[9] I. Kramosil and J. Michalek, Fuzzy metric and statistical metric spaces, Kybernetica. 11 (1975), 326-334.

[10] A. K. Mirmostafaee and M. S. Moslehian, Fuzzy almost quadratic functions, Results Math. 52 (2008), 161-177.

[11] A. K. Mirmostafaee and M. S. Moslehian, Fuzzy version of Hyers-Ulam-Rassias theorem, Fuzzy Sets Syst. 159 (2008), 720-729.

[12] T. M. Rassias, On the stability of the linear mapping in Banach spaces, Proc. Amer. Math. Soc. 72 (1978), 297-300.

[13] S. M. Ulam, A Collection of Mathematial Problems, Interscience Tracts in Pure and Applied Mathematics,no.8, Interscience, New York, NY, USA, 1964.

Department of Mathematics Education

Dankook University

Yongin 448-701, Republic of Korea

E-mail: gilhan@dankook.ac.kr 